CRYSTALLOGRAPHIC COMMUNICATIONS

ISSN 2056-9890

Received 8 June 2018

Accepted 12 June 2018

Edited by M. Weil, Vienna University of Technology, Austria

Keywords: crystal structure; hydrogen bonding; azopyridine.

CCDC reference: 1848833

Supporting information: this article has supporting information at journals.iucr.org/e

\section{Crystal structure of 2-[2-(pyridin-3-yl)diazen-1- yl]aniline}

\author{
Morten K. Peters, ${ }^{a}$ Christian Näther ${ }^{b}$ and Rainer Herges ${ }^{a}$ * \\ aOtto-Diels-Institut für Anorganische Chemie, Christian-Albrechts-Universität Kiel, Otto-Hahn-Platz 4, D-24098 Kiel, \\ Germany, and ${ }^{\mathbf{b}}$ Institut für Anorganische Chemie, Christian-Albrechts-Universität Kiel, Max-Eyth Str. 2, D-24118 Kiel, \\ Germany. ${ }^{*}$ Correspondence e-mail: rherges@oc.uni-kiel.de
}

The crystal structure of the title compound, $\mathrm{C}_{11} \mathrm{H}_{10} \mathrm{~N}_{4}$, comprises molecules in a trans conformation for which all the atoms are located in general positions. The six-membered rings are coplanar and this arrangement might be stabilized by intramolecular $\mathrm{N}-\mathrm{H} \cdots \mathrm{N}$ hydrogen bonding. In the crystal, the molecules are linked into helical chains parallel to the $b$ axis via $\mathrm{N}-\mathrm{H} \cdots \mathrm{N}$ hydrogen bonding. The molecular packing shows a herringbone-like pattern along the $a$ axis. Comparison of the X-ray powder diffraction with that calculated from single crystal data proves that a pure crystalline phase was obtained and UV-Vis measurements reveal that only the trans isomer is present.

\section{Chemical context}

Azobenzenes are among the most frequently used photochromic compounds with numerous applications in different fields being reported (Szymański et al., 2013; Merino \& Ribagorda, 2012; Kay et al., 2007). Moreover, azobenzenes are easily accessible, and their photochromic functions are quite reliable. The stretched trans isomer is usually the thermodynamically stable conformation. Upon irradiation with UV light, the bent cis isomer is formed. This cis conformation switches back to the trans isomer either upon irradiation with visible light or thermochemically (Hartley, 1937). A highly important variation of azobenzenes are azopyridines, as pyridines coordinate to various metals, e.g. nickel (Thies et al., 2010; Dommaschk et al., 2015c). Thus, azopyridines can be used as switchable ligands. In this context, we have reported an approach to switch the spin state of azopyridine-functionalized Ni-porphyrins (Thies et al., 2011, 2012; Venkataramani et al., 2011; Dommaschk et al., 2015a,b). Aiming at further functionalization of azopyridines and in view of applications as molecular spin switches, we have synthesized 2-[2-(pyridin3 -yl)diazen-1-yl]aniline and report here its molecular and crystal structure.

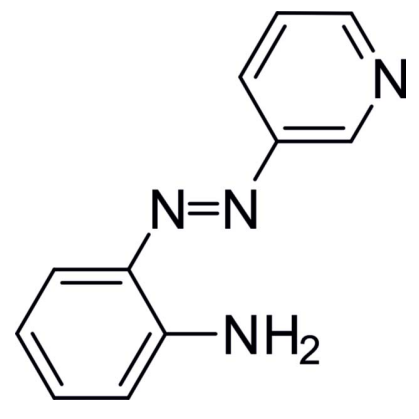


Table 1

Hydrogen-bond geometry $\left(\AA,^{\circ}\right)$.

\begin{tabular}{lllll}
\hline$D-\mathrm{H} \cdots A$ & $D-\mathrm{H}$ & $\mathrm{H} \cdots A$ & $D \cdots A$ & $D-\mathrm{H} \cdots A$ \\
\hline $\mathrm{N} 4-\mathrm{H} 1 N 4 \cdots \mathrm{N} 2$ & $0.881(16)$ & $2.066(15)$ & $2.6922(14)$ & $127.3(12)$ \\
$\mathrm{N} 4-\mathrm{H} 2 N 4 \cdots \mathrm{N}{ }^{\mathrm{i}}$ & $0.904(16)$ & $2.163(16)$ & $3.0274(14)$ & $159.7(14)$ \\
\hline
\end{tabular}

Symmetry code: (i) $-x+\frac{1}{2}, y-1,-z+\frac{3}{2}$.

\section{Structural commentary}

The crystal structure of the title compound comprises 2-[2(pyridin-3-yl)diazen-1-yl]aniline molecules, located on general positions, adopting a trans-conformation with a $\mathrm{C} 1-\mathrm{N} 2-$ N3-C6 torsion angle of $-179.80(8)^{\circ}$, which corresponds to the energetically favored arrangement (Fig. 1). The two sixmembered rings are coplanar [the maximum deviation from the least-squares plane for all non-H atoms is 0.0569 (9) $\AA$ for $\mathrm{N} 4]$ and the dihedral angle between the ring planes is $0.11(8)^{\circ}$. The amino $\mathrm{H}$ atoms are also in the plane of the adjacent benzene ring. There is an intramolecular $\mathrm{N}-\mathrm{H} \cdots \mathrm{N}$ hydrogen bond between one of the amino $\mathrm{H}$ atoms and one nitrogen atom of the azo group with an $\mathrm{N} \cdots \mathrm{H}$ distance of 2.066 (15) $\AA$ and an $\mathrm{N}-\mathrm{H} \cdots \mathrm{N}$ angle of 127.30 (12) (Table 1). Even if this corresponds to a weak interaction, it might stabilize the planar arrangement.

\section{Supramolecular features}

In the crystal structure of the title compound, the molecules are linked into chains along the $b$-axis direction via $\mathrm{N}-\mathrm{H} \cdots \mathrm{N}$ hydrogen bonds between the amino hydrogen atom that is not involved in intramolecular hydrogen bonding and one of the nitrogen atoms of the azo group (Fig. 2, top). The $\mathrm{N} \cdots \mathrm{H}$ distance amounts to 2.163 (16) $\AA$ and the $\mathrm{N}-\mathrm{H} \cdots \mathrm{N}$ angle of 159.7.14 (12) $)^{\circ}$ is slightly bent, indicating that this is a relatively strong interaction (Table 1). The dihedral angle between the pyridine ring that carries the acceptor $\mathrm{N}$ atom and the aminophenyl moiety of a neighbouring molecule that carries the donor group is $66.12(8)^{\circ}$. Therefore, the molecules exhibit a helical arrangement along the chain (Fig. 2, top). The chains are closely packed in such a way that each chain is surrounded by eight neighboring chains (Fig. 2, bottom). The molecules

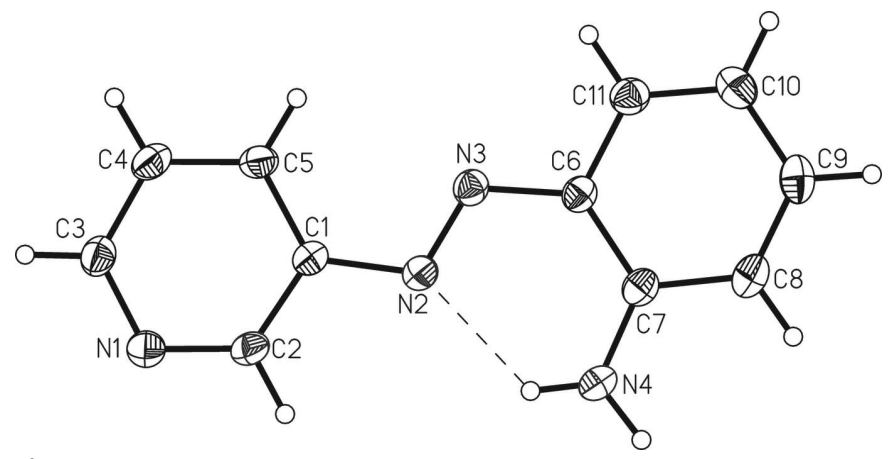

Figure 1

Molecular structure of the title compound with labeling and displacement elliposids drawn at the $50 \%$ probability level. The intramolecular $\mathrm{N}-$ $\mathrm{H} \cdots \mathrm{N}$ hydrogen bond is shown with dashed lines.

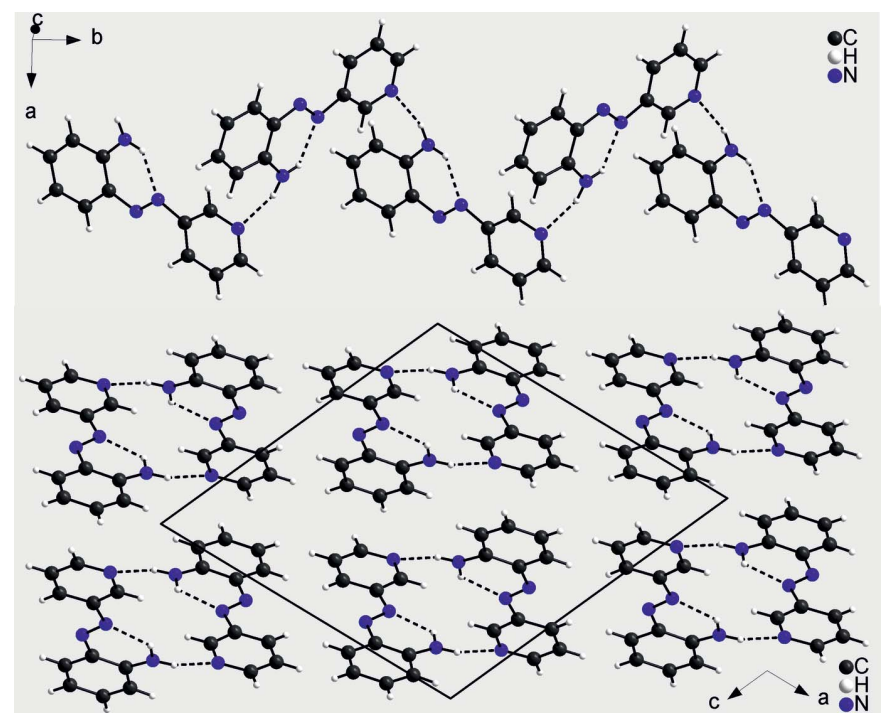

Figure 2

Crystal structure of the title compound showing a chain (top) and a view along the $b$ axis (bottom). Intra- and intermolecular hydrogen bonds are indicated by dashed lines.

exhibit a herringbone-like pattern along the $a$ axis (Fig. 3) in which the pyridine and benzene rings of adjacent molecules are perfectly coplanar. The distance between the ring planes is $3.462 \AA$ and the centroid-centroid distance is 3.8040 (7) $\AA$, indicating $\pi-\pi$ interactions between the chains.

\section{Database survey}

According to a search of the Cambridge Structural Database (CSD; Groom et al., 2016), 3-azopyridine molecules substi-

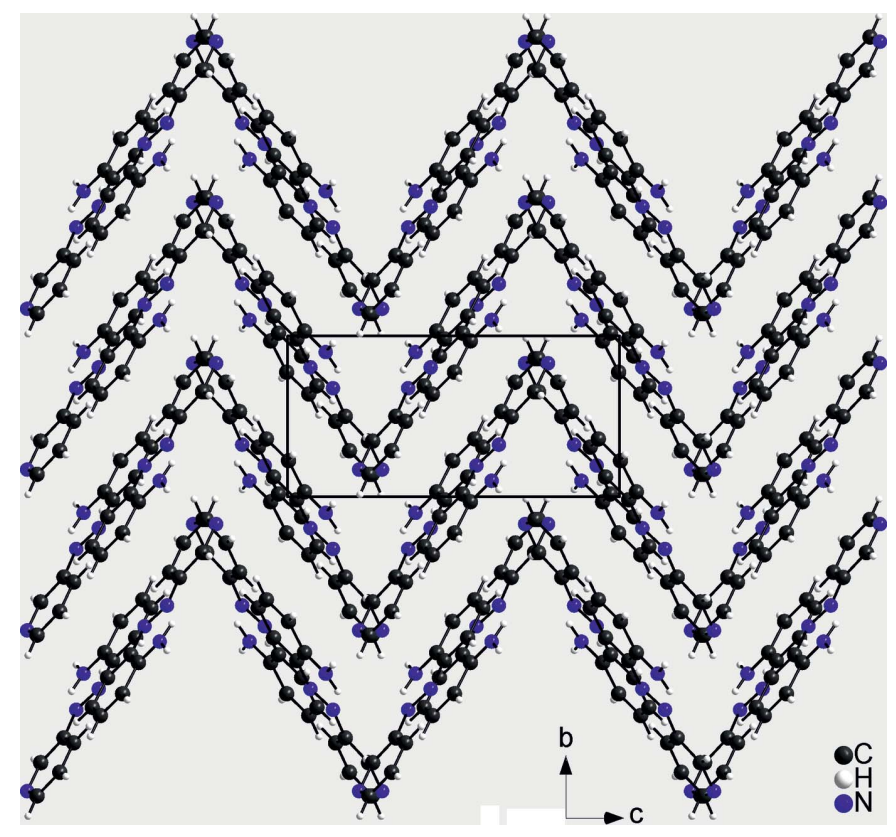

Figure 3

Crystal structure of the title compound in a view along the $a$ axis. 
tuted with an amino group in the ortho-position are unknown. However, one ortho-substituted phenyl-azopyridine was reported, viz. 2,6-diamino-3-[(2-carboxymethyl)phenylazo]pyridine (Tan et al., 2010). 4-Aminophenyl-azopyridines such as $N, N$-diethyl-4-[(E)-(pyridine-3-yl)diazenyl]aniline (Draguta et al., 2015) and N,N-dimethyl-4-(pyridine-3yldiazenyl)aniline (Draguta et al., 2013) are also known. Furthermore, structure reports on 2-azopyridine molecules that are substituted in the ortho-position, such as 5-[(5-bromo2-pyridyl)azo]-2,4-toluenediamine (Jinzi et al., 1984) and 5-[(3,5-dibromo-2-pyridyl)azo]-2,4-diaminotoluene (Kailiang et al., 1985), have been published. Other azo compounds, substituted similarly to the title molecule, are highly important for coordination chemistry, which is shown in their crystal structures (Maiti et al., 2001, 2003; Pratihar et al., 2005, 2007).

\section{Synthesis and crystallization}

The synthesis of the title compound can be performed in two steps.

(i) Synthesis of 3-(2-acetanilide)azopyridine: 2-nitroacetanilide $(4.00 \mathrm{~g}, 29.0 \mathrm{mmol})$ was dissolved in ethanol $(150 \mathrm{ml})$. An aqueous solution of ammonium chloride $(2.01 \mathrm{~g}$, $37.7 \mathrm{mmol}$ in $15 \mathrm{ml}$ ) was added. The mixture was warmed up to $313 \mathrm{~K}$ until the dispersion changed into a clear solution. After cooling to room temperature, zinc dust $(4.93 \mathrm{~g}$, $75.3 \mathrm{mmol}$ ) was added and the mixture was stirred for $1 \mathrm{~h}$ at $333 \mathrm{~K}$. After filtration, the filtrate was poured into an aqueous ice-cooled iron(III) chloride solution (hexaaqua complex, $5.40 \mathrm{~g}, 20.3 \mathrm{mmol}$ in $150 \mathrm{ml}$ ) whereby a green solid precipitated. After $15 \mathrm{~min}$ of stirring, the solid was filtered off and washed with water. The crude product was a mixture of 2-nitrosoacetanilide and starting material, which was used for azo condensation without further purification. 3-Aminopyridine $(800 \mathrm{mg}, 8.51 \mathrm{mmol})$ was dissolved in a mixture of pyridine $(25 \mathrm{ml})$ and aqueous sodium hydroxide $(5 \mathrm{ml}, 25 \%)$. The crude product of 2-nitrosoacetanilide dissolved in pyridine $(30 \mathrm{ml})$ was added to the solution containing the 3aminopyridine. The reaction mixture was stirred for $1 \mathrm{~h}$ at $353 \mathrm{~K}$ and overnight at room temperature. After addition of dichloromethane $(200 \mathrm{ml})$ the phases were separated. The organic layer was washed with water twice and dried over sodium sulfate. The solvent was removed under reduced pressure. The crude product was purified by column chromatography (ethyl ester $/ n$-hexane, $R_{\mathrm{f}}=0.16$ ). The product was obtained as an orange solid. Yield: $200 \mathrm{mg}(0.83 \mathrm{mmol}, 10 \%)$.

${ }^{1} \mathrm{H}$ NMR $(500 \mathrm{MHz}, 300 \mathrm{~K}, \mathrm{CDCl} 3): \delta=9.88(s, \mathrm{br}, 1 \mathrm{H}, \mathrm{N}-$ $\mathrm{H}), 9.11\left(d,{ }^{4} J=2.4 \mathrm{~Hz}, 1 \mathrm{H}\right), 8.71\left(d d,{ }^{3} J=4.7 \mathrm{~Hz},{ }^{4} J=1.5 \mathrm{~Hz}\right)$, $8.66\left(d, b r,{ }^{3} J=8.3 \mathrm{~Hz}, 1 \mathrm{H}\right), 8.07\left(d d d,{ }^{3} J=8.2 \mathrm{~Hz},{ }^{4} J=2.4 \mathrm{~Hz}\right.$, $\left.{ }^{4} J=1.5 \mathrm{~Hz}, 1 \mathrm{H}\right), 7.83\left(d d,{ }^{3} J=8.1 \mathrm{~Hz},{ }^{4} J=1.4 \mathrm{~Hz} 1 \mathrm{H}\right), 7.51-$ $7.44(m, 2 \mathrm{H}), 7.16\left(t d,{ }^{3} J=8.1 \mathrm{~Hz},{ }^{4} J=1.3 \mathrm{~Hz} 1 \mathrm{H}\right), 2.26(s, 3 \mathrm{H})$ ppm. ${ }^{13} \mathrm{C}$ NMR $(150 \mathrm{MHz}, 300 \mathrm{~K}, \mathrm{CDCl} 3): \delta=168.3,151.9$, 147.7, 146.3, 138.9, 136.4, 133.8, 127.3, 124.1, 123.4, 120.6, 120.4, $25.2 \mathrm{ppm}$. HRMS (EI): $\mathrm{m} / z[M]^{+}$calculated for $\mathrm{C}_{13} \mathrm{H}_{12} \mathrm{~N}_{4} \mathrm{O}$ : 240.10111, found: 240.10136 .
Table 2

Experimental details.

\begin{tabular}{|c|c|}
\hline \multicolumn{2}{|l|}{ Crystal data } \\
\hline Chemical formula & $\mathrm{C}_{11} \mathrm{H}_{10} \mathrm{~N}_{4}$ \\
\hline$M_{\mathrm{r}}$ & 198.23 \\
\hline Crystal system, space group & Monoclinic, $P 2 / n$ \\
\hline Temperature (K) & 200 \\
\hline$a, b, c(\AA)$ & $13.2798(8), 5.9792(3), 13.4130(9)$ \\
\hline$\beta\left(^{\circ}\right)$ & $113.046(7)$ \\
\hline$V\left(\AA^{3}\right)$ & $980.03(11)$ \\
\hline$Z$ & 4 \\
\hline Radiation type & Мo $K \alpha$ \\
\hline$\mu\left(\mathrm{mm}^{-1}\right)$ & 0.09 \\
\hline Crystal size $(\mathrm{mm})$ & $0.20 \times 0.15 \times 0.15$ \\
\hline \multicolumn{2}{|l|}{ Data collection } \\
\hline Diffractometer & Stoe IPDS1 \\
\hline $\begin{array}{l}\text { No. of measured, independent and } \\
\text { observed }[I>2 \sigma(I)] \text { reflections }\end{array}$ & $8233,2137,1731$ \\
\hline$R_{\text {int }}$ & 0.073 \\
\hline$(\sin \theta / \lambda)_{\max }\left(\AA^{-1}\right)$ & 0.639 \\
\hline \multicolumn{2}{|l|}{ Refinement } \\
\hline$R\left[F^{2}>2 \sigma\left(F^{2}\right)\right], w R\left(F^{2}\right), S$ & $0.039,0.113,1.04$ \\
\hline No. of reflections & 2137 \\
\hline No. of parameters & 145 \\
\hline $\mathrm{H}$-atom treatment & $\begin{array}{l}\mathrm{H} \text { atoms treated by a mixture of } \\
\text { independent and constrained } \\
\text { refinement }\end{array}$ \\
\hline$\Delta \rho_{\max }, \Delta \rho_{\min }\left(\mathrm{e} \AA^{-3}\right)$ & $0.21,-0.18$ \\
\hline
\end{tabular}

Computer programs: X-AREA (Stoe, 2008), SHELXS97 and XP (Sheldrick, 2008), SHELXL2014 (Sheldrick, 2015), DIAMOND (Brandenburg, 2014) and publCIF (Westrip, 2010).

(ii) Synthesis of 2-[2-(pyridin-3-yl)diazen-1-yl]aniline: 3-(2acetanilide)azopyridine (640 mg, $2.66 \mathrm{mmol}$ ) was dissolved in methanol $(50 \mathrm{ml})$. A sodium hydroxide solution $(5 \mathrm{ml}, 30 \%)$ was added and stirred for $6 \mathrm{~h}$ at $343 \mathrm{~K}$. 2-[2-(Pyridin-3yl)diazen-1-yl]aniline precipitated and was filtered off. The solid was washed with water. Recrystallization from acetone gave orange single crystals. Yield: $520 \mathrm{mg}(2.63 \mathrm{mmol}, 99 \%)$.

${ }^{1} \mathrm{H}$ NMR $\left(500 \mathrm{MHz}, 300 \mathrm{~K}, \mathrm{CDCl}_{3}\right): \delta=9.08\left(d d,{ }^{4} \mathrm{~J}=\right.$ $\left.2.4 \mathrm{~Hz},{ }^{5} J=0.8 \mathrm{~Hz}, 1 \mathrm{H}\right), 8.63\left(d d,{ }^{3} J=4.7 \mathrm{~Hz},{ }^{4} J=1.6 \mathrm{~Hz}, 1 \mathrm{H}\right)$, $8.08\left(d d d,{ }^{3} J=8.2 \mathrm{~Hz},{ }^{4} J=2.4 \mathrm{~Hz},{ }^{4} J=1.6 \mathrm{~Hz}, 1 \mathrm{H}\right), 7.84\left(d d,{ }^{3} \mathrm{~J}\right.$ $\left.=8.1 \mathrm{~Hz},{ }^{4} \mathrm{~J}=1.4 \mathrm{~Hz}, 1 \mathrm{H}\right), 7.41\left(d d d,{ }^{3} J=8.2 \mathrm{~Hz},{ }^{3} J=4.7 \mathrm{~Hz},{ }^{5} \mathrm{~J}\right.$ $=0.8 \mathrm{~Hz}, 1 \mathrm{H}), 7.24\left(d d d,{ }^{3} J=8.3 \mathrm{~Hz},{ }^{3} J=7.1 \mathrm{~Hz},{ }^{4} J=1.4 \mathrm{~Hz}\right.$, $1 \mathrm{H}), 6.83\left(d d d,{ }^{3} J=8.1 \mathrm{~Hz},{ }^{3} J=7.1 \mathrm{~Hz},{ }^{4} J=1.3 \mathrm{~Hz}, 1 \mathrm{H}\right), 6.77$ $\left(d d,{ }^{3} J=8.3 \mathrm{~Hz},{ }^{4} J=1.3 \mathrm{~Hz}, 1 \mathrm{H}\right), 6.01(s, b r, 2 \mathrm{H}, \mathrm{N}-\mathrm{H}) \mathrm{ppm}$. ${ }^{13} \mathrm{C}$ NMR $\left(150 \mathrm{MHz}, 300 \mathrm{~K}, \mathrm{CDCl}_{3}\right): \delta=150.6,148.3,146.51$, 143.0, 137.1, 133.1, 128.5, 126.4, 123.9, 117.5, $117.1 \mathrm{ppm} . \mathrm{MS}$ (EI, TOF): $m / z(\%)=199[M]^{+}, 198[M], 120\left[M-\mathrm{C}_{5} \mathrm{H}_{4} \mathrm{~N}\right]^{+}$, $92\left[M-\mathrm{C}_{6} \mathrm{H}_{6} \mathrm{~N}_{2}\right]^{+}$.

Comparison of the experimental X-ray powder diffraction pattern with that calculated from single crystal data proves that the title compound was obtained as a pure phase (see Fig. $\mathrm{S} 1$ in the supporting information). The UV-Vis spectrum shows the strong $\pi \rightarrow \pi^{*}$ band of the trans conformation (Fig. S2 in the supporting information). If the sample is exposed to light of $365 \mathrm{~nm}$, no isomerization into the cis conformer is observed, and the sample starts to decompose. However, conversion of the amino to an amide group will probably restore the photochromic properties. 


\section{Refinement}

Crystal data, data collection and structure refinement details are summarized in Table 2 . The $\mathrm{C}-\mathrm{H}$ hydrogen atoms were located in a difference map but were positioned with idealized geometry and refined with $U_{\text {iso }}(\mathrm{H})=1.2 U_{\text {eq }}(\mathrm{C}, \mathrm{N})$ using a riding model with $\mathrm{C}_{\text {aromatic }}-\mathrm{H}=0.95 \AA$. The $\mathrm{N}-\mathrm{H}$ hydrogen atoms were located in a difference map and were freely refined.

\section{Acknowledgements}

We thank Professor Dr Wolfgang Bensch for access to his experimental facilities.

\section{Funding information}

The authors gratefully acknowledge financial support from the Deutsche Forschungsgesellschaft within the Sonderforschungsbereich 677 .

\section{References}

Brandenburg, K. (2014). DIAMOND. Crystal Impact GbR, Bonn, Germany.

Dommaschk, M., Näther, C. \& Herges, R. (2015a). J. Org. Chem. 80, 8496-8500.

Dommaschk, M., Peters, M., Gutzeit, F., Schütt, C., Näther, C., Sönnichsen, F. D., Tiwari, S., Riedel, C., Boretius, S. \& Herges, R. (2015b). J. Am. Chem. Soc. 137, 7552-7555.

Dommaschk, M., Thoms, V., Schütt, C., Näther, C., Puttreddy, R., Rissanen, K. \& Herges, R. (2015c). Inorg. Chem. 54, 9390-9392.

Draguta, S., Fonari, M. S., Leonova, E. \& Timofeeva, T. (2015). J. Mol. Struct. 1098, 206-215.

Draguta, S., Leonova, E., Fokina, M., Denisyuk, I. \& Timofeeva, T. V. (2013). Acta Cryst. E69, o1280.
Groom, C. R., Bruno, I. J., Lightfoot, M. P. \& Ward, S. C. (2016). Acta Cryst. B72, 171-179.

Hartley, G. S. (1937). Nature, 140, 281-281.

Jinzi, Q., Jiaxing, Y., Haifu, F., Kailiang, S. \& Shenghua, H. (1984). Acta Scient. Nat. Univ. Sunyatseni, 17, 2.

Kailiang, S., Shenghua, H., Jinzi, Q., Jiaxing, Y. \& Haifu, F. (1985). Chem. J. Chin. Univ. 6, 573.

Kay, E. R., Leigh, D. A. \& Zerbetto, F. (2007). Angew. Chem. Int. Ed. 46, 72-191.

Maiti, N., Dirghangi, B. K. \& Chattopadhyay, S. (2003). Polyhedron, 22, 3109-3113.

Maiti, N., Pal, S. \& Chattopadhyay, S. (2001). Inorg. Chem. 40, 2204 2205.

Merino, E. \& Ribagorda, M. (2012). Beilstein J. Org. Chem. 8, 10711090.

Pratihar, J. L., Maiti, N. \& Chattopadhyay, S. (2005). Inorg. Chem. 44, 6111-6114.

Pratihar, J. L., Shee, B., Pattanayak, P., Patra, D., Bhattacharyya, A., Puranik, V. G., Hung, C. H. \& Chattopadhyay, S. (2007). Eur. J. Inorg. Chem. pp. 4272-4281.

Sheldrick, G. M. (2008). Acta Cryst. A64, 112-122.

Sheldrick, G. M. (2015). Acta Cryst. A71, 3-8.

Stoe (2008). $X$-AREA. Stoe \& Cie, Darmstadt, Germany.

Szymański, W., Beierle, J. M., Kistemaker, H. A. V., Velema, W. A. \& Feringa, B. L. (2013). Chem. Rev. 113, 6114-6178.

Tan, X., Xie, X., Chen, J. \& Zhan, S. (2010). Inorg. Chem. Commun. 13, 1455-1458.

Thies, S., Bornholdt, C., Köhler, F., Sönnichsen, F. D., Näther, C., Tuczek, F. \& Herges, R. (2010). Chem. Eur. J. 16, 10074-10083.

Thies, S., Sell, H., Bornholdt, C., Schütt, C., Köhler, F., Tuczek, F. \& Herges, R. (2012). Chem. Eur. J. 18, 16358-16368.

Thies, S., Sell, H., Schütt, C., Bornholdt, C., Näther, C., Tuczek, F. \& Herges, R. (2011). J. Am. Chem. Soc. 133, 16243-16250.

Venkataramani, S., Jana, U., Dommaschk, M., Sönnichsen, F. D., Tuczek, F. \& Herges, R. (2011). Science, 331, 445-448.

Westrip, S. P. (2010). J. Appl. Cryst. 43, 920-925. 


\section{supporting information}

Acta Cryst. (2018). E74, 1013-1016 [https://doi.org/10.1107/S2056989018008605]

\section{Crystal structure of 2-[2-(pyridin-3-yl)diazen-1-yl]aniline}

\section{Morten K. Peters, Christian Näther and Rainer Herges}

Computing details

Data collection: $X$-AREA (Stoe, 2008); cell refinement: $X$-AREA (Stoe, 2008); data reduction: $X$-AREA (Stoe, 2008); program(s) used to solve structure: SHELXS97 (Sheldrick, 2008); program(s) used to refine structure: SHELXL2014 (Sheldrick, 2015); molecular graphics: XP (Sheldrick, 2008) and DIAMOND (Brandenburg, 2014); software used to prepare material for publication: publCIF (Westrip, 2010).

2-[2-(Pyridin-3-yl)diazen-1-yl]aniline

Crystal data

$\mathrm{C}_{11} \mathrm{H}_{10} \mathrm{~N}_{4}$

$M_{r}=198.23$

Monoclinic, $P 2 / n$

$a=13.2798(8) \AA$

$b=5.9792(3) \AA$

$c=13.4130(9) \AA$

$\beta=113.046(7)^{\circ}$

$V=980.03(11) \AA^{3}$

$Z=4$

Data collection

Stoe IPDS-1 diffractometer

Phi scans

8233 measured reflections

2137 independent reflections

1731 reflections with $I>2 \sigma(I)$

Refinement

Refinement on $F^{2}$

Least-squares matrix: full

$R\left[F^{2}>2 \sigma\left(F^{2}\right)\right]=0.039$

$w R\left(F^{2}\right)=0.113$

$S=1.04$

2137 reflections

145 parameters

0 restraints

Hydrogen site location: mixed
$F(000)=416$

$D_{\mathrm{x}}=1.344 \mathrm{Mg} \mathrm{m}^{-3}$

Mo $K \alpha$ radiation, $\lambda=0.71073 \AA$

Cell parameters from 8233 reflections

$\theta=3.3-27.0^{\circ}$

$\mu=0.09 \mathrm{~mm}^{-1}$

$T=200 \mathrm{~K}$

Block, orange

$0.20 \times 0.15 \times 0.15 \mathrm{~mm}$

$R_{\text {int }}=0.073$

$\theta_{\max }=27.0^{\circ}, \theta_{\min }=3.3^{\circ}$

$h=-16 \rightarrow 16$

$k=-7 \rightarrow 7$

$l=-17 \rightarrow 17$

$\mathrm{H}$ atoms treated by a mixture of independent and constrained refinement

$w=1 /\left[\sigma^{2}\left(F_{\mathrm{o}}^{2}\right)+(0.0675 P)^{2}+0.0778 P\right]$ where $P=\left(F_{\mathrm{o}}^{2}+2 F_{\mathrm{c}}^{2}\right) / 3$

$(\Delta / \sigma)_{\max }<0.001$

$\Delta \rho_{\max }=0.21 \mathrm{e} \AA^{-3}$

$\Delta \rho_{\min }=-0.18 \mathrm{e} \AA^{-3}$

Extinction correction: SHELXL2014

(Sheldrick, 2015),

$\mathrm{Fc}^{*}=\mathrm{kFc}\left[1+0.001 \mathrm{xFc}^{2} \lambda^{3} / \sin (2 \theta)\right]^{-1 / 4}$

Extinction coefficient: 0.054 (14) 


\section{Special details}

Geometry. All esds (except the esd in the dihedral angle between two 1.s. planes) are estimated using the full covariance matrix. The cell esds are taken into account individually in the estimation of esds in distances, angles and torsion angles; correlations between esds in cell parameters are only used when they are defined by crystal symmetry. An approximate (isotropic) treatment of cell esds is used for estimating esds involving l.s. planes.

Fractional atomic coordinates and isotropic or equivalent isotropic displacement parameters $\left(\AA^{2}\right)$

\begin{tabular}{lllll}
\hline & $x$ & $y$ & $z$ & $U_{\text {iso }} / U_{\text {eq }}$ \\
\hline C1 & $0.42109(8)$ & $0.49909(17)$ & $0.67373(8)$ & $0.0197(3)$ \\
C2 & $0.40530(8)$ & $0.65338(19)$ & $0.74372(9)$ & $0.0240(3)$ \\
H2 & 0.3449 & 0.6313 & 0.7638 & $0.029^{*}$ \\
N1 & $0.46934(7)$ & $0.83068(16)$ & $0.78456(8)$ & $0.0264(3)$ \\
C3 & $0.55368(9)$ & $0.85966(19)$ & $0.75476(9)$ & $0.0252(3)$ \\
H3 & 0.5997 & 0.9861 & 0.7817 & $0.030^{*}$ \\
C4 & $0.57689(9)$ & $0.71309(19)$ & $0.68624(9)$ & $0.0261(3)$ \\
H4 & 0.6382 & 0.7387 & 0.6680 & $0.031^{*}$ \\
C5 & $0.51034(9)$ & $0.53033(18)$ & $0.64487(9)$ & $0.0236(3)$ \\
H5 & 0.5248 & 0.4281 & 0.5979 & $0.028^{*}$ \\
N2 & $0.34292(7)$ & $0.32432(14)$ & $0.63669(7)$ & $0.0218(2)$ \\
N3 & $0.35904(7)$ & $0.19490(15)$ & $0.56895(7)$ & $0.0216(2)$ \\
C6 & $0.28594(8)$ & $0.01793(17)$ & $0.52757(8)$ & $0.0205(3)$ \\
C7 & $0.19205(8)$ & $-0.03083(18)$ & $0.55077(8)$ & $0.0221(3)$ \\
C8 & $0.13128(9)$ & $-0.22461(19)$ & $0.50278(9)$ & $0.0272(3)$ \\
H8 & 0.0687 & -0.2617 & 0.5171 & $0.033^{*}$ \\
C9 & $0.16052(9)$ & $-0.3602(2)$ & $0.43605(9)$ & $0.0294(3)$ \\
H9 & 0.1186 & -0.4903 & 0.4059 & $0.035^{*}$ \\
C10 & $0.25156(10)$ & $-0.30942(19)$ & $0.41168(9)$ & $0.0291(3)$ \\
H10 & 0.2705 & -0.4022 & 0.3642 & $0.035^{*}$ \\
C11 & $0.31265(9)$ & $-0.12381(19)$ & $0.45757(9)$ & $0.0247(3)$ \\
H11 & 0.3747 & -0.0897 & 0.4418 & $0.030^{*}$ \\
N4 & $0.15968(9)$ & $0.09982(18)$ & $0.61468(9)$ & $0.0308(3)$ \\
H1N4 & $0.2024(12)$ & $0.210(2)$ & $0.6510(12)$ & $0.034(4)^{*}$ \\
H2N4 & $0.1081(13)$ & $0.045(3)$ & $0.6365(13)$ & $0.045(4)^{*}$ \\
& & & &
\end{tabular}

Atomic displacement parameters $\left(\AA^{2}\right)$

\begin{tabular}{lllllll}
\hline & $U^{11}$ & $U^{22}$ & $U^{33}$ & $U^{12}$ & $U^{13}$ & $U^{23}$ \\
\hline $\mathrm{C} 1$ & $0.0178(5)$ & $0.0203(5)$ & $0.0213(5)$ & $0.0009(4)$ & $0.0080(4)$ & $0.0027(4)$ \\
$\mathrm{C} 2$ & $0.0186(5)$ & $0.0268(6)$ & $0.0293(5)$ & $0.0009(4)$ & $0.0125(4)$ & $-0.0011(4)$ \\
$\mathrm{N} 1$ & $0.0232(5)$ & $0.0264(5)$ & $0.0313(5)$ & $0.0005(4)$ & $0.0125(4)$ & $-0.0045(4)$ \\
$\mathrm{C} 3$ & $0.0219(5)$ & $0.0222(5)$ & $0.0309(6)$ & $-0.0024(4)$ & $0.0098(4)$ & $-0.0007(4)$ \\
$\mathrm{C} 4$ & $0.0221(5)$ & $0.0284(6)$ & $0.0321(6)$ & $-0.0021(4)$ & $0.0152(4)$ & $0.0021(5)$ \\
$\mathrm{C} 5$ & $0.0242(5)$ & $0.0251(6)$ & $0.0262(6)$ & $0.0005(4)$ & $0.0150(4)$ & $0.0000(4)$ \\
$\mathrm{N} 2$ & $0.0211(4)$ & $0.0221(5)$ & $0.0240(5)$ & $-0.0010(3)$ & $0.0106(4)$ & $0.0000(4)$ \\
$\mathrm{N} 3$ & $0.0220(4)$ & $0.0196(5)$ & $0.0238(5)$ & $-0.0001(3)$ & $0.0097(4)$ & $0.0019(4)$ \\
C6 & $0.0203(5)$ & $0.0198(5)$ & $0.0211(5)$ & $0.0008(4)$ & $0.0078(4)$ & $0.0032(4)$ \\
C7 & $0.0208(5)$ & $0.0240(6)$ & $0.0208(5)$ & $-0.0005(4)$ & $0.0073(4)$ & $0.0037(4)$
\end{tabular}




\begin{tabular}{lllllll} 
C8 & $0.0226(5)$ & $0.0300(6)$ & $0.0274(6)$ & $-0.0050(4)$ & $0.0080(4)$ & $0.0028(5)$ \\
C9 & $0.0279(6)$ & $0.0238(6)$ & $0.0301(6)$ & $-0.0046(4)$ & $0.0045(5)$ & $-0.0016(5)$ \\
C10 & $0.0304(6)$ & $0.0255(6)$ & $0.0296(6)$ & $0.0034(5)$ & $0.0099(5)$ & $-0.0029(5)$ \\
C11 & $0.0236(5)$ & $0.0249(6)$ & $0.0267(6)$ & $0.0025(4)$ & $0.0110(4)$ & $0.0023(4)$ \\
N4 & $0.0291(5)$ & $0.0351(6)$ & $0.0362(6)$ & $-0.0100(4)$ & $0.0214(4)$ & $-0.0073(5)$ \\
\hline
\end{tabular}

Geometric parameters $\left(\AA,{ }^{o}\right)$

\begin{tabular}{|c|c|c|c|}
\hline $\mathrm{C} 1-\mathrm{C} 2$ & $1.3895(15)$ & $\mathrm{C} 6-\mathrm{C} 11$ & $1.4094(15)$ \\
\hline $\mathrm{C} 1-\mathrm{C} 5$ & $1.3959(14)$ & $\mathrm{C} 6-\mathrm{C} 7$ & $1.4283(14)$ \\
\hline $\mathrm{C} 1-\mathrm{N} 2$ & $1.4186(13)$ & $\mathrm{C} 7-\mathrm{N} 4$ & $1.3484(15)$ \\
\hline $\mathrm{C} 2-\mathrm{N} 1$ & $1.3350(14)$ & $\mathrm{C} 7-\mathrm{C} 8$ & $1.4148(15)$ \\
\hline $\mathrm{C} 2-\mathrm{H} 2$ & 0.9500 & $\mathrm{C} 8-\mathrm{C} 9$ & $1.3716(17)$ \\
\hline $\mathrm{N} 1-\mathrm{C} 3$ & $1.3397(13)$ & $\mathrm{C} 8-\mathrm{H} 8$ & 0.9500 \\
\hline $\mathrm{C} 3-\mathrm{C} 4$ & $1.3892(15)$ & $\mathrm{C} 9-\mathrm{C} 10$ & $1.4035(17)$ \\
\hline $\mathrm{C} 3-\mathrm{H} 3$ & 0.9500 & C9-H9 & 0.9500 \\
\hline $\mathrm{C} 4-\mathrm{C} 5$ & $1.3781(15)$ & $\mathrm{C} 10-\mathrm{C} 11$ & $1.3703(16)$ \\
\hline $\mathrm{C} 4-\mathrm{H} 4$ & 0.9500 & $\mathrm{C} 10-\mathrm{H} 10$ & 0.9500 \\
\hline $\mathrm{C} 5-\mathrm{H} 5$ & 0.9500 & $\mathrm{C} 11-\mathrm{H} 11$ & 0.9500 \\
\hline $\mathrm{N} 2-\mathrm{N} 3$ & $1.2738(12)$ & $\mathrm{N} 4-\mathrm{H} 1 \mathrm{~N} 4$ & $0.881(16)$ \\
\hline $\mathrm{N} 3-\mathrm{C} 6$ & $1.3958(13)$ & $\mathrm{N} 4-\mathrm{H} 2 \mathrm{~N} 4$ & $0.904(16)$ \\
\hline $\mathrm{C} 2-\mathrm{C} 1-\mathrm{C} 5$ & $117.88(10)$ & $\mathrm{C} 11-\mathrm{C} 6-\mathrm{C} 7$ & $119.42(10)$ \\
\hline $\mathrm{C} 2-\mathrm{C} 1-\mathrm{N} 2$ & $116.26(9)$ & $\mathrm{N} 4-\mathrm{C} 7-\mathrm{C} 8$ & $119.78(10)$ \\
\hline $\mathrm{C} 5-\mathrm{C} 1-\mathrm{N} 2$ & $125.86(10)$ & $\mathrm{N} 4-\mathrm{C} 7-\mathrm{C} 6$ & $122.88(10)$ \\
\hline $\mathrm{N} 1-\mathrm{C} 2-\mathrm{C} 1$ & $124.34(10)$ & $\mathrm{C} 8-\mathrm{C} 7-\mathrm{C} 6$ & $117.34(10)$ \\
\hline $\mathrm{N} 1-\mathrm{C} 2-\mathrm{H} 2$ & 117.8 & $\mathrm{C} 9-\mathrm{C} 8-\mathrm{C} 7$ & $121.61(10)$ \\
\hline $\mathrm{C} 1-\mathrm{C} 2-\mathrm{H} 2$ & 117.8 & $\mathrm{C} 9-\mathrm{C} 8-\mathrm{H} 8$ & 119.2 \\
\hline $\mathrm{C} 2-\mathrm{N} 1-\mathrm{C} 3$ & $116.94(9)$ & $\mathrm{C} 7-\mathrm{C} 8-\mathrm{H} 8$ & 119.2 \\
\hline $\mathrm{N} 1-\mathrm{C} 3-\mathrm{C} 4$ & $122.94(10)$ & $\mathrm{C} 8-\mathrm{C} 9-\mathrm{C} 10$ & $120.92(10)$ \\
\hline $\mathrm{N} 1-\mathrm{C} 3-\mathrm{H} 3$ & 118.5 & $\mathrm{C} 8-\mathrm{C} 9-\mathrm{H} 9$ & 119.5 \\
\hline $\mathrm{C} 4-\mathrm{C} 3-\mathrm{H} 3$ & 118.5 & $\mathrm{C} 10-\mathrm{C} 9-\mathrm{H} 9$ & 119.5 \\
\hline $\mathrm{C} 5-\mathrm{C} 4-\mathrm{C} 3$ & $119.55(10)$ & $\mathrm{C} 11-\mathrm{C} 10-\mathrm{C} 9$ & $118.89(10)$ \\
\hline $\mathrm{C} 5-\mathrm{C} 4-\mathrm{H} 4$ & 120.2 & $\mathrm{C} 11-\mathrm{C} 10-\mathrm{H} 10$ & 120.6 \\
\hline $\mathrm{C} 3-\mathrm{C} 4-\mathrm{H} 4$ & 120.2 & $\mathrm{C} 9-\mathrm{C} 10-\mathrm{H} 10$ & 120.6 \\
\hline $\mathrm{C} 4-\mathrm{C} 5-\mathrm{C} 1$ & $118.35(10)$ & $\mathrm{C} 10-\mathrm{C} 11-\mathrm{C} 6$ & $121.81(10)$ \\
\hline $\mathrm{C} 4-\mathrm{C} 5-\mathrm{H} 5$ & 120.8 & $\mathrm{C} 10-\mathrm{C} 11-\mathrm{H} 11$ & 119.1 \\
\hline $\mathrm{C} 1-\mathrm{C} 5-\mathrm{H} 5$ & 120.8 & $\mathrm{C} 6-\mathrm{C} 11-\mathrm{H} 11$ & 119.1 \\
\hline $\mathrm{N} 3-\mathrm{N} 2-\mathrm{C} 1$ & $113.14(9)$ & $\mathrm{C} 7-\mathrm{N} 4-\mathrm{H} 1 \mathrm{~N} 4$ & $119.2(9)$ \\
\hline $\mathrm{N} 2-\mathrm{N} 3-\mathrm{C} 6$ & $117.34(9)$ & $\mathrm{C} 7-\mathrm{N} 4-\mathrm{H} 2 \mathrm{~N} 4$ & $117.8(10)$ \\
\hline $\mathrm{N} 3-\mathrm{C} 6-\mathrm{C} 11$ & $113.80(9)$ & $\mathrm{H} 1 \mathrm{~N} 4-\mathrm{N} 4-\mathrm{H} 2 \mathrm{~N} 4$ & $119.8(14)$ \\
\hline $\mathrm{N} 3-\mathrm{C} 6-\mathrm{C} 7$ & $126.78(10)$ & & \\
\hline $\mathrm{C} 5-\mathrm{C} 1-\mathrm{C} 2-\mathrm{N} 1$ & $0.57(16)$ & $\mathrm{N} 3-\mathrm{C} 6-\mathrm{C} 7-\mathrm{N} 4$ & $2.90(17)$ \\
\hline $\mathrm{N} 2-\mathrm{C} 1-\mathrm{C} 2-\mathrm{N} 1$ & $-178.53(10)$ & $\mathrm{C} 11-\mathrm{C} 6-\mathrm{C} 7-\mathrm{N} 4$ & $-178.27(10)$ \\
\hline $\mathrm{C} 1-\mathrm{C} 2-\mathrm{N} 1-\mathrm{C} 3$ & $0.34(16)$ & $\mathrm{N} 3-\mathrm{C} 6-\mathrm{C} 7-\mathrm{C} 8$ & $-177.68(9)$ \\
\hline $\mathrm{C} 2-\mathrm{N} 1-\mathrm{C} 3-\mathrm{C} 4$ & $-1.12(16)$ & $\mathrm{C} 11-\mathrm{C} 6-\mathrm{C} 7-\mathrm{C} 8$ & $1.15(15)$ \\
\hline $\mathrm{N} 1-\mathrm{C} 3-\mathrm{C} 4-\mathrm{C} 5$ & $0.96(17)$ & $\mathrm{N} 4-\mathrm{C} 7-\mathrm{C} 8-\mathrm{C} 9$ & $179.05(10)$ \\
\hline
\end{tabular}




$\begin{array}{llll}\mathrm{C} 3-\mathrm{C} 4-\mathrm{C} 5-\mathrm{C} 1 & 0.01(16) & \mathrm{C} 6-\mathrm{C} 7-\mathrm{C} 8-\mathrm{C} 9 & -0.39(15) \\ \mathrm{C} 2-\mathrm{C} 1-\mathrm{C} 5-\mathrm{C} 4 & -0.72(15) & \mathrm{C} 7-\mathrm{C} 8-\mathrm{C} 9-\mathrm{C} 10 & -0.88(17) \\ \mathrm{N} 2-\mathrm{C} 1-\mathrm{C} 5-\mathrm{C} 4 & 178.28(10) & \mathrm{C} 8-\mathrm{C} 9-\mathrm{C} 10-\mathrm{C} 11 & 1.36(17) \\ \mathrm{C} 2-\mathrm{C} 1-\mathrm{N} 2-\mathrm{N} 3 & 176.88(9) & \mathrm{C} 9-\mathrm{C} 10-\mathrm{C} 11-\mathrm{C} 6 & -0.56(17) \\ \mathrm{C} 5-\mathrm{C} 1-\mathrm{N} 2-\mathrm{N} 3 & -2.14(15) & \mathrm{N} 3-\mathrm{C} 6-\mathrm{C} 11-\mathrm{C} 10 & 178.29(9) \\ \mathrm{C} 1-\mathrm{N} 2-\mathrm{N} 3-\mathrm{C} 6 & -179.80(8) & \mathrm{C} 7-\mathrm{C} 6-\mathrm{C} 11-\mathrm{C} 10 & -0.69(16) \\ \mathrm{N} 2-\mathrm{N} 3-\mathrm{C} 6-\mathrm{C} 11 & -176.92(9) & \mathrm{C} 1-\mathrm{N} 2-\mathrm{N} 3-\mathrm{C} 6 & -179.80(8) \\ \mathrm{N} 2-\mathrm{N} 3-\mathrm{C} 6-\mathrm{C} 7 & 1.96(15) & & \end{array}$

Hydrogen-bond geometry $\left(A,{ }^{\circ}\right)$

\begin{tabular}{lllll}
\hline$D-\mathrm{H} \cdots A$ & $D-\mathrm{H}$ & $\mathrm{H} \cdots A$ & $D \cdots A$ & $D-\mathrm{H} \cdots A$ \\
\hline $\mathrm{N} 4-\mathrm{H} 1 N 4 \cdots \mathrm{N} 2$ & $0.881(16)$ & $2.066(15)$ & $2.6922(14)$ & $127.3(12)$ \\
$\mathrm{N} 4-\mathrm{H} 2 N 4 \cdots \mathrm{N} 1^{\mathrm{i}}$ & $0.904(16)$ & $2.163(16)$ & $3.0274(14)$ & $159.7(14)$
\end{tabular}

Symmetry code: (i) $-x+1 / 2, y-1,-z+3 / 2$. 